\title{
Light control of surface-bulk coupling by terahertz vibrational coherence in a topological insulator
}

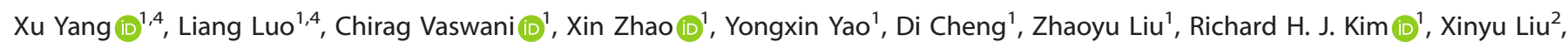
Malgorzata Dobrowolska-Furdyna ${ }^{2}$, Jacek K. Furdyna ${ }^{2}$, llias E. Perakis ${ }^{3}$, Caizhuang Wang ${ }^{1}$, Kaiming $\mathrm{Ho}^{1}$ and Jigang Wang (iD)

The demand for disorder-tolerant quantum logic and spin electronics can be met by generating and controlling dissipationless spin currents protected by topology. Dirac fermions with helical spin-locking surface transport offer a way of achieving such a goal. Yet, surface-bulk coupling can lead to strong Dirac electron scattering with bulk carriers and phonons as well as impurities, assisted by such dissipative channel, which results in "topological breakdown". Here, we demonstrate that coherent lattice vibrations periodically driven by a single-cycle terahertz $(\mathrm{THz})$ pulse can significantly suppress such dissipative channel in topological insulators. This is achieved by reducing the phase space in the bulk available for Dirac fermion scattering into during coherent lattice oscillations in $\mathrm{Bi}_{2} \mathrm{Se}_{3}$. This light-induced suppression manifests as a remarkable transition exclusively in surface transport, absent for bulk, above the THz electric fields for driving coherent phonons, which prolongs the surface transport lifetime. These results, together with simulations, identify the critical role of spin-orbit coupling for the "phase space contraction" mechanism that suppresses the surface-bulk coupling. Imposing vibrational quantum coherence into topological states of matter may become a universal light control principle for reinforcing the symmetry-protected helical transport.

npj Quantum Materials (2020)5:13; https://doi.org/10.1038/s41535-020-0215-7

\section{INTRODUCTION}

Recently, there is increasing evidence that coherently driven states can protect and enhance the stability of quantum systems. A pedagogical example for such dynamic stabilization is the periodically driven Kapitza pendulum ${ }^{1}$ where an inverted, yet stable, orientation can be achieved by a sufficiently high frequency vibration of its pivotal point. Applied to topological protected states (TPS), such as those realized in topological insulators (TIs), ${ }^{2-7}$ coherent lattice vibrations can have direct and profound effects on surface transport of Dirac fermions, via periodic modulation of electronic states, as illustrated in Fig. 1 a. This effect of coherent phonons is in stark contrast to that of incoherent phonons from thermal excitations, which diminishes topological enhancement of surface transport via random lattice scattering, heating, surface-bulk charge transfer and coupling. Ultrafast phononics has been explored as a new avenue to manipulate properties of superconductors, ${ }^{8}$ oxides, ${ }^{9,10}$ semime$\mathrm{tal}^{11}$ and photovoltaic semiconductors. ${ }^{12}$ Although experimental realizations of coherent phonon generation have been explored in $\mathrm{Tls}^{7,13,14}$ the influence of such vibrational coherence on the surface helical transport has not been observed until now.

The prototype $\mathrm{Tl}$, such as $\mathrm{Bi}_{2} \mathrm{Se}_{3}$ exhibits surface charge transport protected by spin-momentum locking, where the spin-orbit interaction connects the direction of the carrier spin to that of its momentum. ${ }^{4,15-17}$ This spin-locking gives rise to coherent transport that manifests as, e.g., lack of current relaxation and prolonged transport lifetime, which is protected against nonmagnetic disorder by symmetry. Here, we explore a nonequilibrium driven TPS, periodically modulated by coherent phonons induced by a noncontact $\mathrm{THz}$ push electric field, as a new control knob for further protecting surface carriers from scattering with residual bulk states and/or thermal phonons. Such
$\mathrm{THz}$ photon driven coherence mechanism complements the spontaneous symmetry protection mechanism in the ground state. However, its observation requires intense $\mathrm{THz}$ pump and $\mathrm{THz}$ conductivity probe spectroscopy measurements ${ }^{18-22}$ of the coherent phonon-dressed TPS, which has never been demonstrated so far.

In this article, we create a periodically driven topologically protected state via coherent lattice vibrations that significantly suppress the surface-bulk coupling in $\mathrm{Bi}_{2} \mathrm{Se}_{3}$. We demonstrate two regimes of post-THz-pump nonequilibrium states, obtained by tuning $E_{\mathrm{THz}}$. In this way we show evidence of nonthermal quantum control of the Dirac fermion transport lifetime $\tau_{\text {Dirac, }}$ by inducing a transition from incoherent carrier heating to a coherent driving regime. This transition exclusively appears in surface transport and is absent in bulk. It occurs above electric field strengths required for observing coherent phonon oscillations. Together with theoretical simulations, our results reveal a "phase space contraction" mechanism assisted by spin-orbit coupling that is responsible for coherent phonon-enabled suppression of the surfacebulk coupling in dressed topological states out of equilibrium.

\section{RESULTS}

We apply a single-cycle $\mathrm{THz}$ pulse $E_{\mathrm{THz}}$ with amplitude up to $\sim 500 \mathrm{kV} \mathrm{cm}^{-1}$, which selectively excites coherent oscillations of $E_{u}^{1}$ phonons, shown in Fig. $1 \mathrm{~b}$. For $E_{\mathrm{THz}}=0$, the real part of the static conductivity, $\sigma_{1}(\omega)$, at the ground state shows a Drude lineshape superimposed to a well-defined oscillator feature centered at $\sim 8 \mathrm{meV}$ as shown in Fig. 1c. This lineshape has been established as arising from joint carrier conductivity responses from both bulk and surface states plus a $E_{u}^{1}$ phonon mode, consistent with prior $\mathrm{THz}$ electrodynamic studies of $\mathrm{Bi}_{2} \mathrm{Se}_{3}{ }^{23}$ For $\mathrm{THz}$ excited states, i.e.,

\footnotetext{
${ }^{1}$ Department of Physics and Astronomy and Ames Laboratory-U.S. DOE, lowa State University, Ames, IA 50011, USA. ${ }^{2}$ Department of Physics, University of Notre Dame, Notre Dame, IN 46556, USA. ${ }^{3}$ Department of Physics, University of Alabama at Birmingham, Birmingham, AL 35294-1170, USA. ${ }^{4}$ These authors contributed equally: Xu Yang, Liang Luo. 凶email: jgwang@ameslab.gov
} 
a

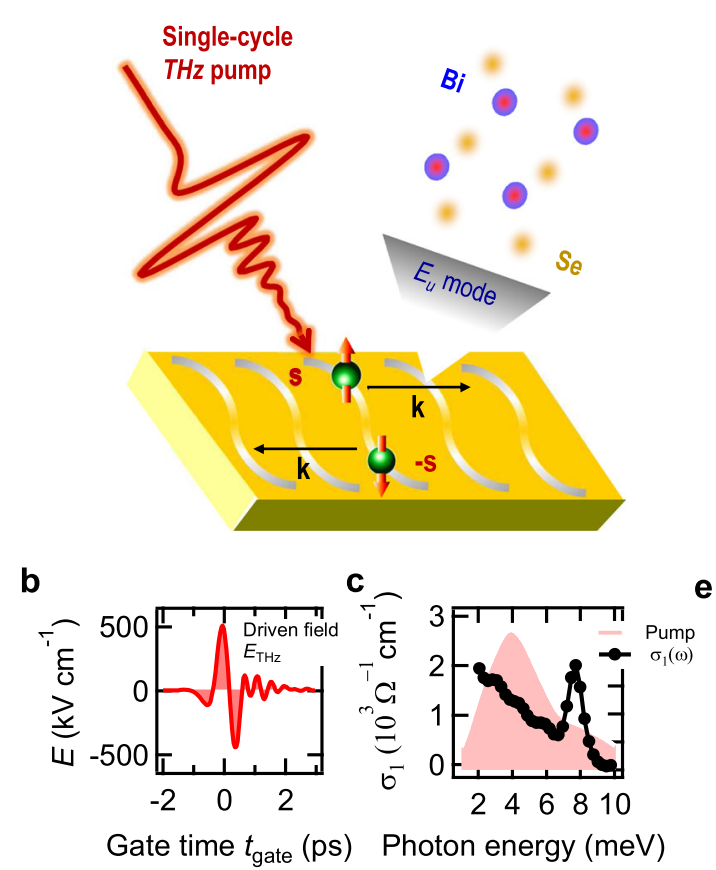

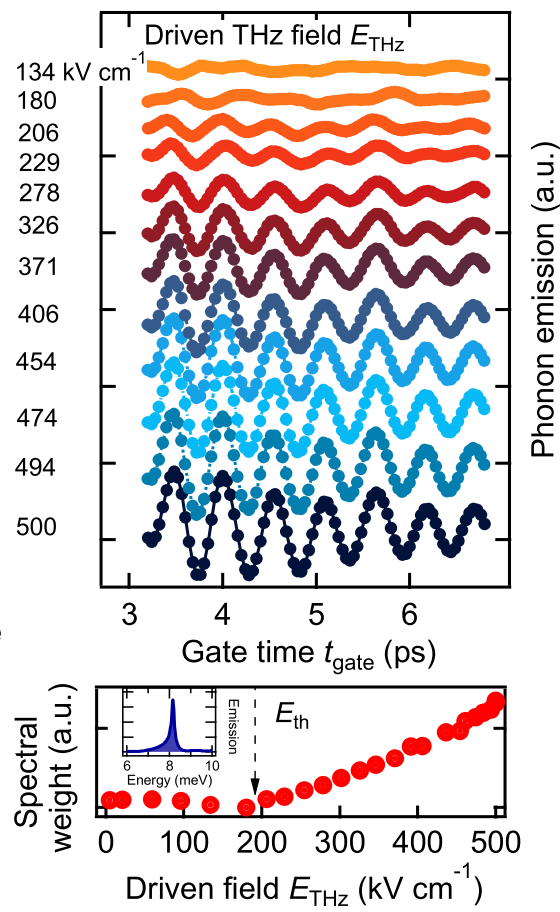

Fig. 1 Vibrational quantum coherence in $\mathrm{Bi}_{2} \mathrm{Se}_{3}$ driven by an intense single-cycle $\mathrm{THz}$ pulse above a threshold electric field. a Experimental schematics of the $\mathrm{THz}$ pump process in $\mathrm{Bi}_{2} \mathrm{Se}_{3}$ film. Intense $\mathrm{THz}$ pulses drive coherent phonon oscillations of the $E_{u}^{1}$ mode that strongly influence surface state transport. b THz electric field profile in the time domain, with peak field as large as $500 \mathrm{kV} \mathrm{cm}^{-1}$ and c broadband spectrum from 1 to $10 \mathrm{meV}$ (pink shade). The static conductivity $\sigma_{1}(\omega)$, shown together in c, exhibits Drude transport behavior superimposed to a bulk $E_{u}^{1}$ phonon mode resonance at $\sim 8 \mathrm{meV}$ (black solid circle). $\mathbf{d}$ The post-pump THz emission for various driving THz electric fields demonstrates a threshold-like behavior clearly observed above $E_{\mathrm{th}} \sim 180 \mathrm{kV} \mathrm{cm}^{-1}$. e Pump electric field dependence of the coherent phonon integrated spectral weight shows a significant increase above threshold $E_{\mathrm{th}}$. Inset: Corresponding Fourier spectrum of timedomain $\mathrm{THz}$ emission driven by single-cycle $\mathrm{THz}$ pump with E-field of $500 \mathrm{kV} \mathrm{cm}^{-1}$.

$E_{\mathrm{THz}} \neq 0$, the spectral-temporal characteristics of the complex conductivity response functions are measured by our $\mathrm{THz}$ pump-THz probe conductivity spectroscopy that allows us to differentiate surface from bulk contributions as shown in ref. ${ }^{23}$. In this way we measure the transport lifetimes of Dirac currents, $\tau_{\text {Dirac, }}$ and bulk ones, $\tau_{\text {Bulk, }}$ which reveals the interaction between topological states and bulk coherent phonons.

We study 50 quintuple layers of n-type $\mathrm{Bi}_{2} \mathrm{Se}_{3}$ with bulk insulating bandgap $\sim 310 \mathrm{meV}$ and Fermi energy $\sim 60 \mathrm{meV}$ above the conduction band edge. This $\mathrm{Bi}_{2} \mathrm{Se}_{3}$ film is grown by molecular beam epitaxy on top of a $0.5 \mathrm{~mm}$ thick sapphire substrate with caxis perpendicular to surface. The sample is cooled down to $5 \mathrm{~K}$ in a He flow cryostat in order to maximize the surface responses by reducing thermal phonons.

Figure $1 \mathrm{~d}$ shows phonon emission after $\mathrm{THz}$ pump at $5 \mathrm{~K}$ as a function of electric field $E_{\mathrm{THz}}$ up to $\sim 500 \mathrm{kV} \mathrm{cm}^{-1}$. The broadband $\mathrm{THz}$ pulses used here are centered at $\omega_{\text {pump }} \sim 4 \mathrm{meV}$ (Fig. 1c) and can excite the $E_{u}^{1}$ phonon mode. Interestingly, the phonon emission clearly shows a pronounced multi-cycle oscillation for high driving fields $\sim 180 \mathrm{kV} \mathrm{cm}^{-1}$. The Fourier transform (FT) spectra of these coherent oscillations displays a resonant peak centered at $\sim 8 \mathrm{meV}$, which is consistent with the $E_{u}^{1}$ phonon mode (inset, Fig. 1e). The integrated spectral weight (SW) clearly shows a "threshold-like" build-up at $E_{\mathrm{THz}} \sim 180 \mathrm{kV} \mathrm{cm}^{-1}$ (Fig. 1e). Below $E_{\mathrm{th}}$ $\sim 180 \mathrm{kV} \mathrm{cm}^{-1}$, the coherent phonon THz emission SW is vanishingly small. This nonlinear emergence of the coherent phonon oscillations is most likely due to the multi-photon excitation of coherent $E_{u}^{1}$ phonons $\sim 8 \mathrm{meV}$. The $\mathrm{THz}$ pump spectra is broad, from 2 to $6 \mathrm{meV}$ with center $\sim 4 \mathrm{meV}$ (Fig. 1c). Therefore, the excitation will be a combination of multiple processes that mainly include two- and three-photon nonlinear processes. This is consistent with the fitted data (Supplementary Note 6), which shows a power law with exponent $\sim 2.7$ in the E-field dependence. Most intriguingly, this transition in lattice displacement driven outof-equilibrium distinguishes two different $\mathrm{THz}$ pumping regimes controlled by the $\mathrm{THz}$ electric field: (1) weak pumping can mainly excite incoherent phonons, averaging to zero lattice movement, and mainly accesses a lattice heating regime; (2) intense $\mathrm{THz}$ driving allows the establishment of a macroscopic lattice vibration and accesses a periodically driven TPS. The latter regime gives rise to the observed $\mathrm{THz}$ radiation, as shown in Fig. $1 \mathrm{~d}$. Note that $\mathrm{THz}$ pulses will also excite charge carriers by intraband transitions at surface and bulk because of finite doping.

We characterize the ultrafast $\mathrm{THz}$ conductivity by measuring the $\mathrm{THz}$ fields in the time domain, shown in the inset of Fig. $2 \mathrm{a}$, b. In the inset, we show $E(t)$ after transmission through (i) a reference substrate, $E_{\text {ref }}(t)$ (gray) and (ii) the unexcited sample, $E_{\text {Sample }}(t)$ (blue). We also compare the above to the pump-induced change $\Delta E(t)$ after pump-probe delay $\Delta t_{\mathrm{pp}}=0.5 \mathrm{ps}$ (red, inset). Using FT and Fresnel equations, the full $\mathrm{THz}$ dielectric response is obtained and expressed as the real parts of the conductivity, $\Delta \sigma_{1}(\omega)$, Fig. $2 \mathrm{a}$, and the dielectric function, $\Delta \varepsilon_{1}(\omega)$, Fig. $2 b$. These response functions characterize the change in transport properties of surface Dirac electrons and bulk carriers following $\mathrm{THz}$ driving. We observe a clear bipolar frequency-dependence of $\Delta \sigma_{1}(\omega)$, with transition from strong bleaching $\left(\Delta \sigma_{1}<0\right)$ to enhanced absorption $\left(\Delta \sigma_{1}>0\right)$ for frequencies between 2 and $10 \mathrm{meV}$. We also observe a decrease of $\Delta \varepsilon_{1}$ with increasing probe frequency in the same range. In our previous study of intraband photoexcitation responses in $\mathrm{Bi}_{2} \mathrm{Se}_{3}$, mainly using mid-infrared pulses, ${ }^{23}$ we identified a similar bipolar spectral shape of $\Delta \sigma_{1}(\omega)$ dynamics, which arises from the combined responses due to competing surface and bulk states. There we showed that the above behavior can be used to distinguish the intrinsic transport properties of 

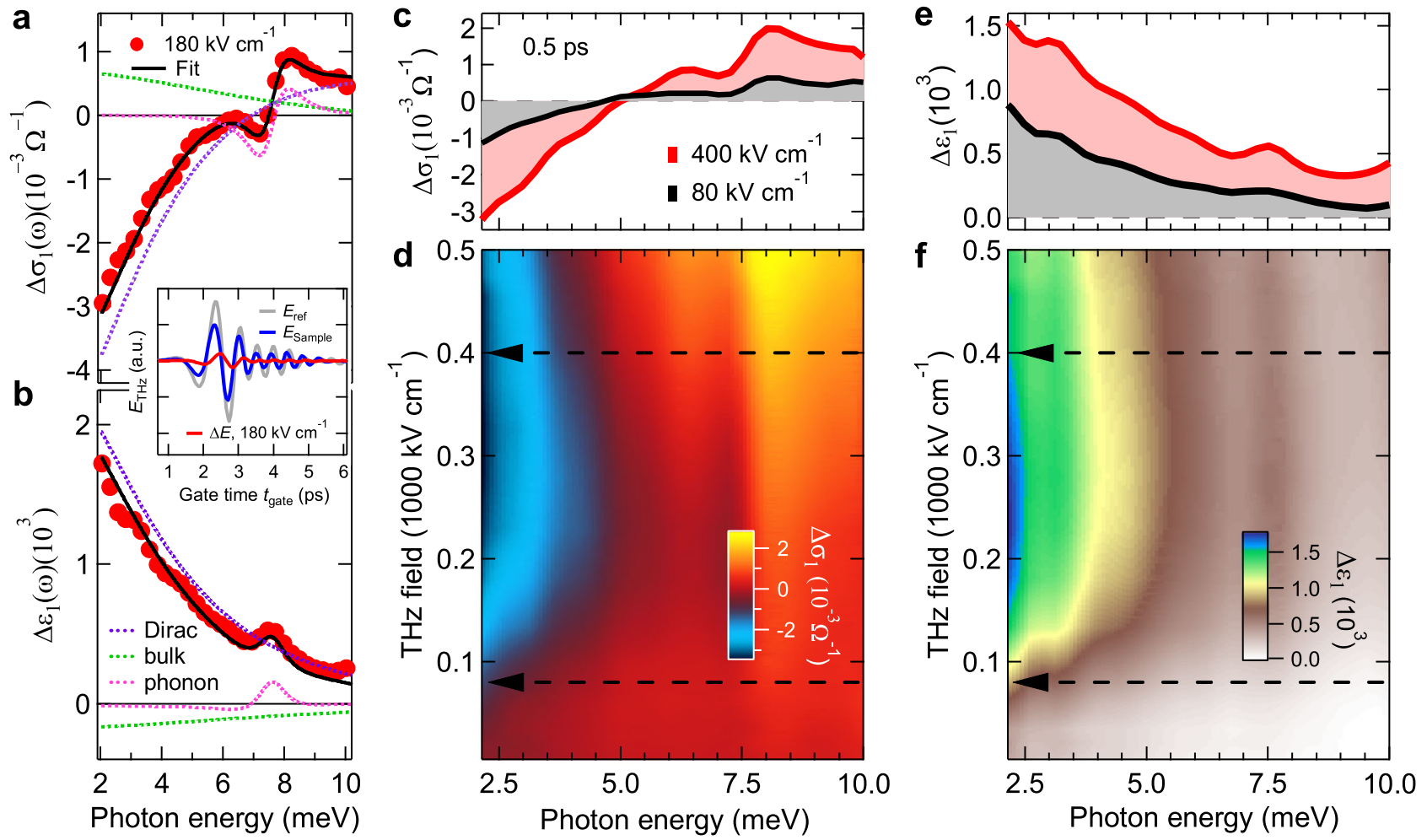

Fig. 2 Frequency dependent dynamics of THz spectra as a function of THz driving field in $\mathbf{B i}_{2} \mathrm{Se}_{3}$. Pump-induced THz spectra, a $\Delta \sigma_{1}(\omega)$ and b $\Delta \varepsilon_{1}(\omega)$, after THz driving with electric field $180 \mathrm{kV} \mathrm{cm}^{-1}$ at $T=5 \mathrm{~K}$. Shown together is the theoretical fitting of the pump-induced THz spectra. $\Delta \sigma_{1}(\omega)$ and $\Delta \varepsilon_{1}(\omega)$ at $180 \mathrm{kV} \mathrm{cm}^{-1}$, which are well reproduced by a composite model consisting of two Drude and one Lorentz oscillator contributions (main text), account for the Dirac fermions on the surface, the bulk carriers, and the $E_{u}^{1}$ phonon at 8 meV, respectively. Inset: Representative time domain THz raw data. The transmitted $\mathrm{E}$ field through reference $E_{\text {ref }}$ (gray line) and sample $E_{\text {Sample }}(\mathrm{blue}$ line), as well

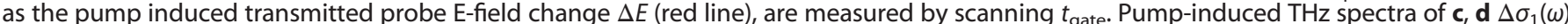
and $\mathbf{e}, \mathbf{f} \Delta \varepsilon_{1}(\omega)$ as a function of THz pumping field at pump-probe delay $\Delta t_{\mathrm{pp}}=0.5 \mathrm{ps}$. $\mathbf{c}$, e The THz spectra at two cuts of the corresponding $2 \mathrm{D}$ plots of $\mathbf{d}$ and $\mathbf{f}$ indicated by the black dashed lines, at driving electric field $E_{\mathrm{THz}}=80$ and $400 \mathrm{kV} \mathrm{cm}^{-1}$, respectively.

Dirac fermions and bulk electrons. The lineshape can also be explained by quantitative simulation as applied below.

The observed spectral lineshapes as a function of pump-probe time delay can be fitted by using a composite model with two Drude terms and one Lorentz oscillator. These three contributions account for the $\mathrm{THz}$ response functions of surface and bulk carriers and phonons, respectively

$$
\begin{aligned}
\tilde{\varepsilon}(\omega) & =\sum_{j=S, B} \tilde{\varepsilon}_{j}^{\text {Drude }}(\omega)+\varepsilon_{\mathrm{ph}}(\omega) \\
& =\varepsilon_{\infty}-\sum_{j=S, \mathrm{~B}} \frac{\left(\omega_{\mathrm{p}}\right)_{j}^{2}}{\omega^{2}+i \omega \gamma_{j}}+\frac{F}{\left(\omega_{0}\right)^{2}-\omega^{2}-i \omega \Gamma} .
\end{aligned}
$$

where $\varepsilon_{\infty}$ is the background electrical permittivity. The plasma frequencies $\omega_{\mathrm{p}}^{2}=n e^{2} / \varepsilon_{0} m^{*}$ are proportional to the densities of surface $\left(n_{\mathrm{S}}\right)$ and bulk $\left(n_{\mathrm{B}}\right)$ free carriers. The subscripts $j=\mathrm{S}$ and $\mathrm{B}$ in the equation stand for surface and bulk states, respectively. $\omega, e$, and $\varepsilon_{0}$ denote the frequency, electron charge, and vacuum electrical permittivity, respectively. $m^{*}$ and $\gamma$ are the effective mass and scattering rates of surface or bulk carriers. The $\mathrm{THz}$ response functions measured in the experiment are well reproduced for all time delays by varying the surface and bulk scattering rates $\gamma_{j}$, the phonon oscillator strength $F$, and the phonon damping coefficient $\Gamma$. At the same time, we keep the surface and bulk carrier densities fixed, consistent with a physical picture of sub-gap photoexcitation that is decoupled from interband transitions. ${ }^{23} \mathrm{~A}$ unique set of model parameters is obtained by simultaneously fitting conductivity and permittivity in the measured broad frequency range. By only varying the surface $\tau_{\mathrm{S}}$ and bulk $\tau_{\mathrm{B}}$ scattering times (i.e., $1 / \gamma$ ), we are able to consistently reproduce the time- dependent experimental results, as shown in Fig. 2a, b, and extract the Dirac (purple line) and bulk (green line) transport properties. This fitting implies that the $\mathrm{THz}$ pumping used here gives rise to much smaller changes of surface and bulk quasiparticle densities as compared to the scattering time changes, unlike for high frequency pumping as discussed in ref. ${ }^{23}$. We can thus separate the intrinsic surface and bulk transport properties in both time and frequency domains.

The distinct spectral-temporal characteristics observed here can be used to characterize how $\mathrm{THz}$ driving influences carrier transport. In Fig. 2c-f, complete 2D false color plots of $\Delta \sigma_{1}$ and $\Delta \varepsilon_{1}$ spectra are presented as a function of $\mathrm{THz}$ pump electric field $E_{\mathrm{THz}}$ up to $500 \mathrm{kV} \mathrm{cm}^{-1}$, at short pump-probe delay $t_{\mathrm{pp}}=0.5 \mathrm{ps}$ after excitation. The pump-induced $\mathrm{THz}$ conductivity spectra $\Delta \sigma_{1}(\omega)$ exhibit a bipolar behavior, demonstrated by the two $\mathrm{THz}$ field-cut spectra in Fig. 2c taken at the black dashed lines in Fig. $2 \mathrm{~d}$. We observe a pump field-dependent nonlinear negative growth and then decay of $\Delta \sigma_{1}(\omega)$ at $5 \mathrm{~K}$. As shown in Fig. 2, the low frequency negative signals from 2 to $4 \mathrm{meV}$ become dominant for driving fields above $\sim 180 \mathrm{kV} \mathrm{cm}^{-1}$ and then progressively decrease with increasing $\mathrm{THz}$ pump field. To analyze this behavior, we fit the $2 \mathrm{D} \mathrm{THz}$ response functions $\Delta \sigma_{1}(\omega)$ and $\Delta \varepsilon_{1}(\omega)$ shown in Fig. $2 d, f$ by using the composite model discussed above. Excellent agreement between this model and the measured transient $\mathrm{THz}$ response is achieved for all $\mathrm{THz}$ pump fluences, similar to the exemplary fitting in Fig. 2a, b. As we discuss next, these results indicate that the transport properties of Dirac fermions arise from two competing effects: coherent phonon periodic modulation and incoherent phonon heating of the Fermi sea. 
4

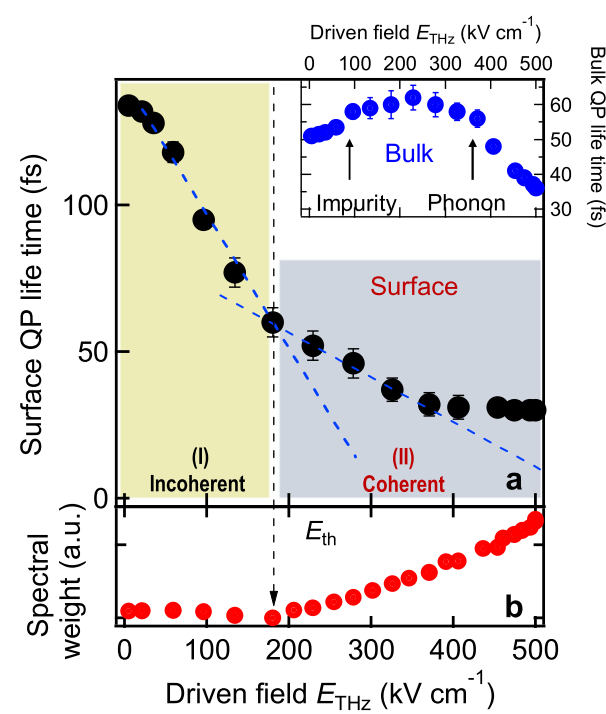

Fig. 3 THz electric field dependence of surface and bulk transport lifetimes in periodically driven $\mathrm{Bi}_{2} \mathrm{Se}_{3}$. a Surface transport lifetime $\tau_{\mathrm{S}}$ showing a transition from an incoherent heating to a coherent driving regime at the threshold $E_{\mathrm{th}} \sim 180 \mathrm{kV} \mathrm{cm}^{-1}$ for emergence of coherent phonon oscillations shown together in b. Above this threshold field, the phonon heating-induced reduction in $\tau_{\mathrm{S}}$ slows down and finally saturates at $\sim 400 \mathrm{kV} \mathrm{cm}^{-1}$. Inset: The bulk transport lifetime $\tau_{\mathrm{B}}$ shows very little change at $E_{\mathrm{th}}$. The error bar indicates the uncertainty from theoretical fitting of the experimental results. c Schematics of surface-bulk scattering in the two $\mathrm{THz}$ excitation regimes marked in a: (1) thermal heating regime by incoherent phonons and (2) coherent driving regime by lattice vibration. The former increases the surface-bulk scattering while the latter suppresses it, thus providing an extra protection for topological transport on the surface.

Figure 3a compares the $\mathrm{THz}$ driving field $\left(E_{\mathrm{THZ}}\right)$ dependence of the transport lifetimes of the surface, $\tau_{S}$, and bulk, $\tau_{B}$, electrons extracted from Fig. $2 d$, f. It shows a clear correlation with the amplitude of coherent phonon emission. $\tau_{\mathrm{s}}$ undergoes a clear transition at $\sim 180 \mathrm{kV} \mathrm{cm}^{-1}$ and saturates at $400 \mathrm{kV} \mathrm{cm}^{-1}$. Below $\sim 180 \mathrm{kV} \mathrm{cm}^{-1}$, there is a reduction in $\tau_{\mathrm{s}}$ with increasing field, marked by the black dashed line, which we assign to increased surface electron scattering due to $\mathrm{THz}$ heating of the Fermi sea and incoherent thermal phonons. Above $\sim 180 \mathrm{kV} \mathrm{cm}^{-1}$, this reduction in $\tau_{\mathrm{S}}$ clearly slows down, which indicates suppression of Dirac fermion scattering compared to what is expected for thermal effects. The observation of a clear transition between two excitation regimes indicates the emergence of additional protection absent in the ground state. This stops further suppression of $\tau_{\mathrm{S}}$ even with increasing $\mathrm{THz}$ field pumping, which finally saturates at the highest $\mathrm{THz}$ field used. The most salient feature is the coincidence between the transition field observed in the Dirac fermion transport lifetime changes and the nonlinear threshold for emergence of coherent phonon oscillations at $E_{\text {th }}$. This coincidence suggests a strong correlation between the two phenomena, i.e, the emergence of prolonged, new nonequilibrium transport lifetimes when the $\mathrm{THz}$ field drives ordered lattice positions via coherent phonon oscillations, instead of disordered lattice positions due to thermal fluctuations. At the same time, the $\mathrm{THz}$ pump field dependence of the bulk transport lifetime $\tau_{\mathrm{B}}$ (inset, Fig. $3 \mathrm{a}$ ) does not show any transition across $E_{\text {th }}$ (Fig. $3 \mathrm{~b}$ ). Instead, $\tau_{B}$ displays an opposite field dependence, i.e., an increase in $\tau_{B}$ at low pump fields followed by a decrease at high pump fields. This behavior can be understood as a transition from impurity- to phonon-dominated scattering with increasing $\mathrm{THz}$ pumping, which is consistent with conventional behaviors seen in the temperature dependence of bulk carrier scattering in a doped

semiconductor. ${ }^{24,25}$ Figure 3 c illustrates our interpretation of these experimental observations as signatures of suppression of the surface-bulk coupling by $\mathrm{THz}$ driving, which leads to a nonequilibrium topological state protected and stabilized further by a new dynamic channel from coherent phonon excitations.

\section{DISCUSSION}

To explore how coherent lattice displacements driven by $E_{\mathrm{THz}}$ influence the topological electronic state, we use first-principles calculations in combination with a microscopic theory of surfacebulk coupling as discussed in details in the Supplementary Note 3. $\mathrm{Bi}_{2} \mathrm{Se}_{3}$ has a rhombohedral structure (space group R-3m) with five atoms in the primitive unit cell (Fig. 4a). These atoms are arranged in a quintuple layered structure, with each layer consisting of five planes of Se2-Bi-Se1-Bi-Se2, with lattice parameters $a=4.142 \AA$ and $c=28.635 \AA$. The details of our first-principles calculation are discussed in the Supplementary Notes 3 and 4. The most important scattering mechanisms of the surface electrons in $\mathrm{Bi}_{2} \mathrm{Se}_{3}$ at low temperature are electron-electron $(e-e)$ and electron-impurity ( $e$-imp) scattering. On the one hand, the $e-e$ scattering rate $\Gamma_{e-e}$ for normal Fermi-liquids is proportional to $T^{2}$. In our THz pump experiment, the surface electronic temperature increases due to intraband scattering after $\mathrm{THz}$ excitation, therefore we expect that $\Gamma_{e-e} \propto\left(E_{\mathrm{THz}}\right)^{4}$ leads to reduced carrier transport lifetimes when heating dominates. On the other hand, the e-imp scattering in the surface is weakly temperaturedependent. Next we explore how the e-imp scattering, assisted by surface-bulk coupling, can be reduced nonthermally by intense $\mathrm{THz}$ driving of coherent phonons that will compete with $\Gamma_{e-e}$ above the threshold as demonstrated in Fig. $3 a$.

The e-imp scattering can proceed via surface-surface (SS) and surface-bulk (SB) channels, whose contribution depends on the available final states, i.e., the surface electron may scatter to either surface or bulk states. According to the microscopic theory of impurity- and phonon-induced SB coupling in Tls, ${ }^{26}$ in n-doped $\mathrm{Bi}_{2} \mathrm{Se}_{3}$ samples with Fermi level at $\sim 60 \mathrm{meV}$ above the conduction band minimum (CBM) similar to ours, the SB scattering channel dominates over the SS one. The SB impurity scattering rate is shown to be proportional to the bulk density of states (DOS) at the Fermi level and is also sensitive to the relative orientation of the surface and bulk orbital pseudospin $\left\langle\tau^{7}\right\rangle{ }^{26}$ Using a model Hamiltonian derived to describe the low-energy electronic structure of $\mathrm{Bi}_{2} \mathrm{Se}_{3}$ up to $\mathrm{O}\left(k^{2}\right)$, ${ }^{27}$ we computed the low energy electronic bands and corresponding surface and bulk expectation values of the orbital pseudospin $\left\langle\tau^{2}\right\rangle$. The results are plotted in Fig. $4 \mathrm{~b}$. It can be seen that, at $60 \mathrm{meV}$ above the CBM, the surface and bulk states have parallel orbital pseudospin orientation. Thereby, the SB scattering rate is enhanced and dominates over other channels, consistent with ref. ${ }^{26}$. Since $\left|\left\langle\tau^{z}\right\rangle_{S}\right| \leq 1,\left|\left\langle\tau^{z}\right\rangle_{B}\right| \simeq 1$, the SB scattering rate of the surface electron at the Fermi level near the conduction band edge takes the following simplified form ${ }^{26}$ (Supplementary Note 4)

$\Gamma_{\mathrm{SB}}^{\mathrm{imp}} \simeq 2 \sum_{a^{\prime} \in \mathrm{B}}\left|g_{\alpha^{\prime}}^{\mathrm{imp}}\right|^{2} U_{\alpha^{\prime}}\left(E_{\mathrm{F}}\right)$,

where the summation includes all bulk bands $a^{\prime} \cdot g_{a^{\prime}}^{\operatorname{imp}}$ is proportional to the effective electron-impurity coupling and is dependent on the overlap between the surface and the $a^{\prime}$ bulk band wavefunctions. $v_{\alpha^{\prime}}\left(E_{\mathrm{F}}\right)$ is the DOS of the bulk band $\alpha^{\prime}$ at the Fermi level. The SB scattering rate $\Gamma_{S B}^{i m p}$ is therefore proportional to the bulk DOS at the Fermi level.

Next we analyze the variation of the bulk DOS at the Fermi level, $v_{\alpha^{\prime}}\left(E_{\mathrm{F}}\right)$, during the lattice vibration under the $E_{u}^{1}$ mode. To determine the lattice dynamics, we calculate the phonon properties with a supercell approach and harmonic approximation using the Phonopy code. ${ }^{28}$ In the calculations, a supercell containing 

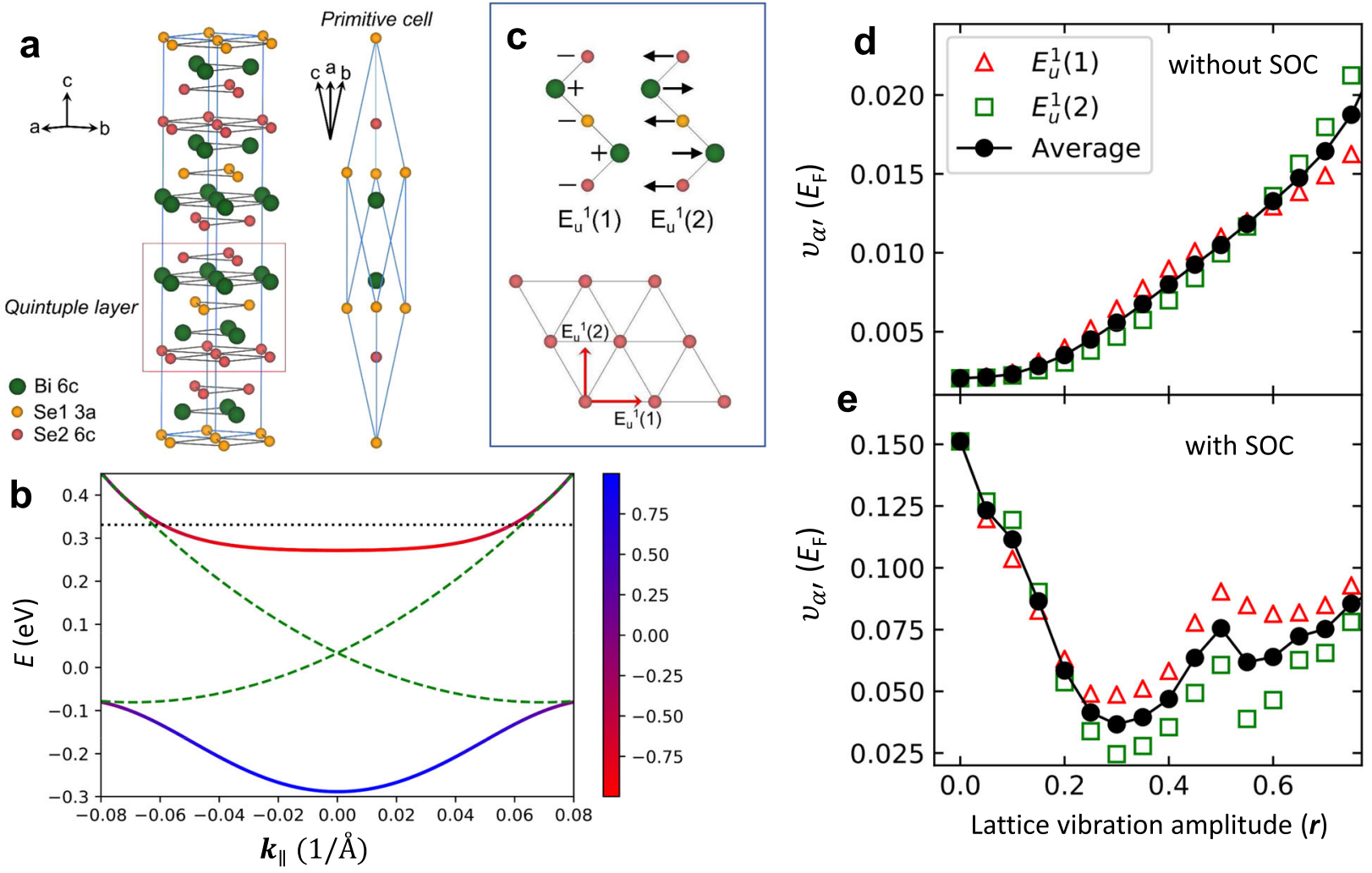

Fig. 4 First-principles calculations, carried out by using DFT and microscopic scattering theory, demonstrate the proposed "phase space contraction" mechanism that suppresses the surface-bulk coupling. a Crystal structure of $\mathrm{Bi}_{2} \mathrm{Se}_{3}$. b Low-energy electronic band structure of the surface (dash lines) and bulk (solid lines) states in $\mathrm{Bi}_{2} \mathrm{Se}_{3}$, calculated using a model Hamiltonian. The color map indicates the value of the orbital pseudospin $\left\langle\tau^{2}\right\rangle$. The Fermi level sits at $60 \mathrm{meV}$ above the conduction band minimum (CBM) and is indicated by the black dotted line. c Schematic representation of the two $E_{u}^{1}$ modes calculated in $\mathrm{Bi}_{2} \mathrm{Se}_{3}$ at $q=0$. The vibration directions of the two inequivalent $E_{u}^{1}$ modes are explained with a hexagonal plane that generally represents all five planes in the $\mathrm{Bi}_{2} \mathrm{Se}_{3}$ quintuple layer. $\mathbf{d}$, e The variation of the bulk DOS at $E_{\mathrm{F}}$ as a function of lattice vibration amplitude (in units of the atomic displacement $r$ ) is calculated from $\mathbf{d}$ GGA and e GGA + SOC.

135 atoms, i.e., $3 \times 3 \times 3$ of the primitive unit cell, and a $k$-point grid of $2 \times 2 \times 2$ are used. The force constants are computed without and with spin-orbit coupling (SOC) using the VASP code. ${ }^{29,30}$ Our results show that the frequency of the $E_{u}^{1}$ mode is $2.57 \mathrm{THz}$ from the GGA calculation, while the GGA + SOC calculation gives $2.30 \mathrm{THz}$, closer to the experimental measurement $(\sim 2.0 \mathrm{THz})$. The directions of the lattice vibrations under the $E_{u}^{1}$ modes are schematically plotted in Fig. 4c, where the two inequivalent $E_{u}^{1}$ modes are illustrated from different views. Note that a hexagonal plane is used to represent all atoms in the quintuple layer without losing generality. The atomic displacement of each layer is determined to be $r=(-0.30,0.40,-0.70$, $0.40,-0.30) \AA$ in the GGA calculation and $r=(-0.31,0.44,-0.65$, $0.44,-0.31) \AA$ in the GGA + SOC calculation, which allows us to track the atomic positions in each plane of the quintuple layer during the harmonic vibration of lattice. Subsequently, the electronic properties are calculated for each snapshot captured during the $E_{u}^{1}$ lattice vibration under the Born-Oppenheimer approximation. Further details are outlined in the Supplementary Notes 3 and 4 and the main results are shown in Fig. $4 d$, e. Here, the bulk DOS at the Fermi level $v_{\alpha^{\prime}}\left(E_{\mathrm{F}}\right)$ is plotted as a function of the lattice vibration amplitude (in units of $r$ ) from GGA and GGA + SOC calculations, respectively, i.e., without (Fig. 4d) and with SOC (Fig. 4e). We note that the results obtained for the two inequivalent $E_{u}^{1}$ modes are not the same, thus an average curve is also computed and plotted in Fig. 4d, e (black solid circles).

The DOS dependence on phonon amplitude is markedly different with or without SOC, which identifies its critical role for determining the phase space available for the SB scattering rate $\Gamma_{\mathrm{SB}}^{\mathrm{imp}}$. Figure $4 \mathrm{~d}$ shows that $v_{\alpha^{\prime}}\left(E_{\mathrm{F}}\right)$ gradually increases in the regular
GGA calculations without SOC, which implies increased Dirac electron scattering. In contrast, the calculations in Fig. 4e with SOC show that $v_{a^{\prime}}\left(E_{\mathrm{F}}\right)$ rapidly decreases for initial decrease of lattice vibrations ( $<0.2 \AA$ from the equilibrium position), and then slowly increases and saturates. The Dirac fermion-impurity scattering rate $\Gamma_{\mathrm{SB}}^{\mathrm{imp}}$, Eq. (2), changes similarly to $v_{\alpha^{\prime}}\left(E_{\mathrm{F}}\right)$ as a function of the lattice vibration amplitude, which is proportional to the $\mathrm{THz}$ pump field. The simulated behavior clearly explains the slow down in $\tau_{\mathrm{S}}$ decrease with this new surface protection channel against bulk scattering and saturation at the highest $\mathrm{THz}$ field used (Fig. 3a). Therefore, the good agreement between the calculated results when SOC is included and the experimental observations clearly underpins the importance of spin-orbit coupling for the "phase space contraction" mechanism that suppresses the surface-bulk coupling in $\mathrm{Bi}_{2} \mathrm{Se}_{3}$ during coherent phonon oscillations. Note that the observed nonequilibrium $\tau_{\mathrm{S}}$ in Fig. 3 a (black circles) is always smaller than the unexcited one mainly due to the unavoidable heating of bulk Fermi sea in highly doped samples like ours. However, the "phase space contraction" mechanism demonstrated in Fig. 4 indicates that further increase in $\tau_{\mathrm{s}}$ can be achieved, e.g., in samples with the doping below the bulk band edge to remove heating effects of bulk carriers. Our data for the bulk conducting state clearly shows the roadmap and it will be interesting to extend the demonstrated coherent-driving scheme to bulk insulating samples and other systems including semimetals and semiconductors to increase coherent transport. ${ }^{11,12,31}$

We expect two major changes with elevated temperature: (1) decrease of coherent phonon amplitude for the same pump field and (2) much stronger surface-bulk coupling, assisted by the population of incoherent phonons. Figure 5 shows these expected 
6
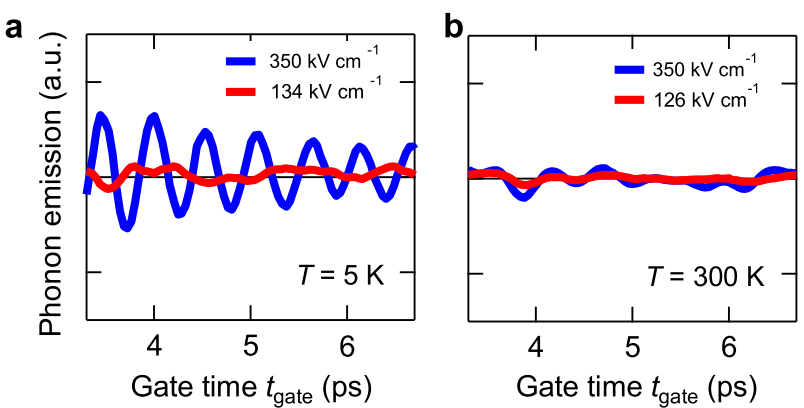

C
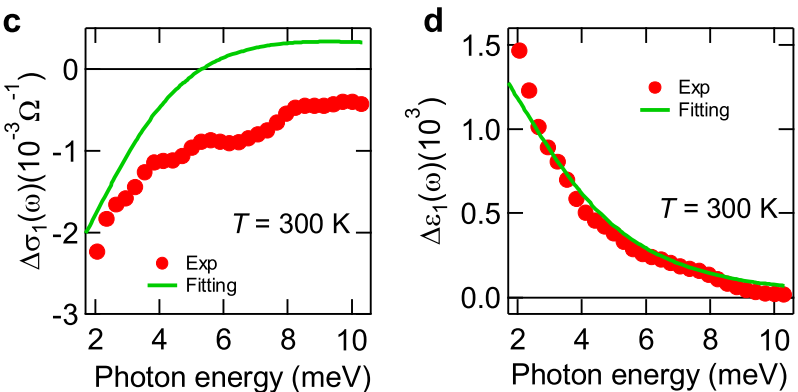

Fig. 5 Enhanced surface-bulk coupling and suppression of the coherent phonon oscillations at high temperature. Comparison of $\mathrm{THz}$ phonon emission at $\mathbf{a} 5 \mathrm{~K}$ and $\mathbf{b} 300 \mathrm{~K}$ after two selected pump fields below and above $E_{\text {th }} \sim 180 \mathrm{kV} \mathrm{cm}^{-1}$. This clearly reveals strong coherent phonon emission at low temperature above $E_{\mathrm{th}}$, while the coherent oscillations are suppressed at $300 \mathrm{~K}$ at the $\mathrm{THz}$ pump fields used. Pump-induced THz spectra of c $\Delta \sigma_{1}(\omega)$ and $\mathbf{d} \Delta \varepsilon_{1}(\omega)$ measured at $300 \mathrm{~K}$. The experimental results cannot be fitted by the composite model (green lines) with fixed surface and bulk densities that works well for low temperature data in Fig. 2a, b.

behaviors. First, Fig. 5a, b compare $\mathrm{THz}$ pump-induced phonon emission at 5 and $300 \mathrm{~K}$ for two selected pump fields, above and below $E_{\text {th }} \sim 180 \mathrm{kV} \mathrm{cm}^{-1}$. One clearly sees coherent phonon oscillation at low temperature above $E_{\mathrm{th}}$, as discussed above. In contrast, the phonon oscillation strength is significantly suppressed at high temperature, due in part to enhanced scattering and thermal population. This result is consistent with our conclusion that the incoherent phonons dominate the $\mathrm{THz}$ driven state at high temperature, unlike for low temperature. This interpretation naturally leads to the conclusion that strong surface-bulk coupling and charge transfer exist at high temperatures as discussed next. Second, THz spectra of $\Delta \sigma_{1}(\omega)$ and $\Delta \varepsilon_{1}(\omega)$ measured at $300 \mathrm{~K}$ (red circles) are shown in Fig. $5 c$, d, which could not be fitted with our composite model (green solid lines) consisting of two Drude contributions with only the scattering rate changes. This is in contrast to the $5 \mathrm{~K}$ data, which are fitted very well with the proposed model. This clearly shows that surface and bulk are strongly coupled by the incoherent phonon with charge transfer in this high temperature case, ${ }^{32}$ which can only be accounted beyond a linear combination of their individual responses.

There are distinguishing features and robust analysis that show the presence of two transport channels, i.e., both surface and bulk, and reliably determine their transport lifetimes, as shown in Fig. 3a. We have addressed them extensively in our prior $\mathrm{THz}$ conductivity study using ultra-broadband, wavelength-selective pumping that extends from mid-IR to near-IR and visible. ${ }^{23}$ Applied to the $\mathrm{THz}$ pump data, for example, one distinguishing feature is the observation of frequency-dependent cooling times that directly distinguish the faster surface channel from the slower bulk one as shown in the Supplementary Note 5. This cannot be explained by a single state with fixed carrier density. The only physical picture that one can propose to consistently explain this unusual behavior is that there are two relaxation channels, i.e., surface and bulk, with different cooling times. ${ }^{23}$

In summery, intense $\mathrm{THz}$-driven coherent phonon modulations provide a previously inaccessible tuning knob for suppressing surface-bulk scattering channels. Our results provide compelling implications of exploring "phononic engineering" of phase space, by modulation of electronic DOS, toward achieving persistent coherent transport in topological device applications.

\section{METHODS}

\section{Experimental schemes}

Broadband quasi-single-cycle $\mathrm{THz}$ pulses were generated by optical rectification in a MgO-doped $\mathrm{LiNbO}_{3}$ crystal using the tilted pulse front method. The crystal was pumped by $2 \mathrm{~W}$ and $35 \mathrm{fs}$ pulses from a Ti:Sapphire based regenerative amplifier with a $1 \mathrm{kHz}$ repetition rate. A portion of the amplifier beam was used to generate $\mathrm{THz}$ probe pulses via optical rectification in a 110-cut $1 \mathrm{~mm}$ ZnTe crystal. The probe THz waveforms were measured using electro-optic sampling in another 110-cut $1 \mathrm{~mm}$ ZnTe crystal. The pump THz electric field was varied using three wire-grid polarizers placed in the THz beam path. The pump THz and probe $\mathrm{THz}$ spot sizes were 1.2 and $0.8 \mathrm{~mm}$, respectively. A $2 \mathrm{~mm}$ aperture was placed in front of the sample to ensure uniform illumination of the probed area. The sample was mounted in an optical cryostat along with a bare substrate which served as a reference. More information can be found in Supplementary Note 1.

\section{Sample preparation}

$\mathrm{The} \mathrm{Bi}_{2} \mathrm{Se}_{3}$ thin film sample, 50 quintuple layers ( $50 \mathrm{~nm}$ thick), is grown by molecular beam epitaxy on a $0.5 \mathrm{~mm}$ thick sapphire substrate. The sample is mounted together with a $0.5 \mathrm{~mm}$ thick pure sapphire substrate, used as a reference, into a cryostat with temperatures down to $T=5 \mathrm{~K}$. Two copper mounts with the same aperture are placed directly in front of the sample and reference to ensure uniform photoexcitation and accurate comparison of their $\mathrm{THz}$ transmission. The sample studied has a Fermi energy $E_{\mathrm{F}}$ $\sim 60 \mathrm{meV}$ from the bulk conduction band edge, as estimated from the measured plasma frequency in the static $\mathrm{THz}$ conductivity spectra. Please note that the exact value of the gap size is not critical for our scheme, nor does it change our conclusion, as long as the interband transition gap in our sample is larger than our pump photon energy $\sim 4 \mathrm{meV}$. Other details can be found in the Supplementary Note 2.

\section{DATA AVAILABILITY}

The data that support the findings of this study are available from the corresponding author upon reasonable request.

Received: 16 October 2019; Accepted: 24 January 2020; Published online: 18 February 2020

\section{REFERENCES}

1. Richards, C. J., Smart, T. J., Jones, P. H. \& Cubero, D. A microscopic Kapitza pendulum. Sci. Rep. 8, 13107 (2018).

2. Hsieh, D. et al. A topological Dirac insulator in a quantum spin Hall phase. Nature 452, 970-974 (2008).

3. Zhang, $\mathrm{H}$. et al. Topological insulators in $\mathrm{Bi}_{2} \mathrm{Se}_{3}, \mathrm{Bi}_{2} \mathrm{Te}_{3}$ and $\mathrm{Sb}_{2} \mathrm{Te}_{3}$ with a single Dirac cone on the surface. Nat. Phys. 5, 438-442 (2009).

4. Hasan, M. Z. \& Kane, C. L. Colloquium: topological insulators. Rev. Mod. Phys. 82, 3045-3067 (2010).

5. Qi, X.-L. \& Zhang, S.-C. The quantum spin Hall effect and topological insulators. Phys. Today 63, 33-38 (2010).

6. Koirala, N. et al. Record surface state mobility and quantum hall effect in topological insulator thin films via interface engineering. Nano Lett. 15, 8245-8249 (2015).

7. Bowlan, P. et al. Probing and controlling terahertz-driven structural dynamics with surface sensitivity. Optica 4, 383-387 (2017).

8. Fausti, D. et al. Light-induced superconductivity in a stripe-ordered cuprate. Science 331, 189-191 (2011).

9. Kozina, M. et al. Terahertz-driven phonon upconversion in $\mathrm{SrTiO}_{3}$. Nat. Phys. 15, 387-392 (2019).

10. $\mathrm{Li}, \mathrm{X}$. et al. Terahertz field-induced ferroelectricity in quantum paraelectric $\mathrm{SrTiO}_{3}$. Science 364, 1079-1082 (2019). 
11. Vaswani, C. et al. Light-Driven Raman Coherence as a Non-Thermal Route to Ultrafast Topology Switching. Preprint at https://arxiv.org/abs/1912.02121 (2019).

12. Liu, L. et al. Single-Cycle Terahertz Driven Quantum Beats Reveal Symmetry-selective Control of Excitonic Fine Structure in Perovskite. Preprint at https://arxiv.org/abs/ 1905.12373 (2019).

13. Weis, M. et al. Ultrafast light-induced coherent optical and acoustic phonons in few quintuple layers of the topological insulator $\mathrm{Bi}_{2} \mathrm{Te}_{3}$. Phys. Rev. B 92, 014301 (2015).

14. Melnikov, A. A. et al. Coherent phonons in a $\mathrm{Bi}_{2} \mathrm{Se}_{3}$ film generated by an intense single-cycle THz pulse. Phys. Rev. B 97, 214304 (2018).

15. Wu, C., Bernevig, B. A. \& Zhang, S.-C. Helical liquid and the edge of quantum spin Hall systems. Phys. Rev. Lett. 96, 106401 (2006).

16. Kane, C. L. \& Mele, E. J. $Z_{2}$ topological order and the quantum spin Hall effect. Phys. Rev. Lett. 95, 146802 (2005).

17. Zyuzin, A. A. \& Burkov, A. A. Topological response in Weyl semimetals and the chiral anomaly. Phys. Rev. B 86, 115133 (2012).

18. Yang, $X$. et al. Terahertz-light quantum tuning of a metastable emergent phase hidden by superconductivity. Nat. Mater. 17, 586-591 (2018).

19. Yang, X. et al. Lightwave-driven gapless superconductivity and forbidden quantum beats by terahertz symmetry breaking. Nat. Photon. 13, 707-713 (2019).

20. Yang, X. et al. Non-equilibrium pair breaking in $\mathrm{Ba}\left(\mathrm{Fe}_{1-x} \mathrm{Co}_{x}\right)_{2} \mathrm{As}_{2}$ superconductors: evidence for formation of photo-induced excitonic spin-densitywave state. Phys. Rev. Lett. 121, 267001 (2018).

21. Yang, X. et al. Ultrafast nonthermal terahertz electrodynamics and possible quantum energy transfer in the $\mathrm{Nb}_{3} \mathrm{Sn}$ superconductor. Phys. Rev. B 99, 094504 (2018).

22. Liu, L. et al. Discovery of Terahertz Second Harmonic Generation from Lightwave Acceleration of Symmetry-Breaking Nonlinear Supercurrents. Preprint at https:// arxiv.org/abs/1912.01676 (2019)

23. Luo, L. et al. Ultrafast manipulation of topologically enhanced surface transport driven by mid-infrared and terahertz pulses in $\mathrm{Bi}_{2} \mathrm{Se}_{3}$. Nat. Commun. 10, 607 (2019).

24. Chattopadhyay, D. \& Queisser, H. J. Electron scattering by ionized impurities in semiconductors. Rev. Mod. Phys. 53, 745-768 (1981).

25. Wang, J. et al. Ultrafast optical manipulation of ferromagnetic order in InMnAs/ GaSb. Proc. IEEE Lester Eastman Conference on High Performance Devices. 307-314 (IEEE, Piscataway, 2002) (2004).

26. Saha, K. \& Garate, I. Theory of bulk-surface coupling in topological insulator films. Phys. Rev. B 90, 245418 (2014).

27. Liu, C.-X. et al. Model Hamiltonian for topological insulators. Phys. Rev. B 82, 045122 (2010).

28. Togo, A. \& Tanaka, I. First principles phonon calculations in materials science. Scr. Mater. 108, 1-5 (2015).

29. Kresse, G. \& Furthmuller, J. Efficiency of ab-initio total energy calculations for metals and semiconductors using a plane-wave basis set. Comput. Mater. Sci. 6, 15-50 (1996).

30. Kresse, G. \& Furthmuller, J. Efficient iterative schemes for ab initio total-energy calculations using a plane-wave basis set. Phys. Rev. B 54, 11169-11186 (1996).

31. Luo, L. et al. Anomalous variations of spectral linewidth in internal excitonic quantum transitions of ultrafast resonantly excited single-walled carbon nanotubes. Phys. Rev. Mater. 3, 026003 (2019).

32. Wang, Y. H. et al. Measurement of intrinsic Dirac Fermion cooling on the surface of the topological insulator $\mathrm{Bi}_{2} \mathrm{Se}_{3}$ using time-resolved and angle-resolved photoemission spectroscopy. Phys. Rev. Lett. 109, 127401 (2012).

\section{ACKNOWLEDGEMENTS}

This work (scientific driver, THz spectroscopy and theory) was supported by the U.S Department of Energy, Office of Basic Energy Science, Division of Materials Sciences and Engineering (Ames Laboratory is operated for the U.S. Department of Energy by lowa State University under Contract No. DE-AC02-07CH11358). Sample development was supported by National Science Foundation DMR 1400432. Terahertz instrument was supported in part by National Science Foundation EECS 1611454. I.E.P. (data analysis) at the University of Alabama, Birmingham was supported by the US Department of Energy, Office of Science, Basic Energy Sciences under award no. DESC0019137.

\section{AUTHOR CONTRIBUTIONS}

X.Y., C.V., and L.L. performed the experimental measurements and collected the data. D.C., Z.L., and R.H.J.K. helped to set up experimental instrumentation. The calculation and some data analysis was done by X.Z., Y.Y., I.E.P., C.Z.W., and K.M.H. Samples were developed by X.L., M.D.; and J.K.F. J.W., and X.Y wrote the paper with help from all authors. J.W. supervised the study.

\section{COMPETING INTERESTS}

The authors declare no competing interests.

\section{ADDITIONAL INFORMATION}

Supplementary information is available for this paper at https://doi.org/10.1038/ s41535-020-0215-7.

Correspondence and requests for materials should be addressed to J.W.

Reprints and permission information is available at http://www.nature.com/ reprints

Publisher's note Springer Nature remains neutral with regard to jurisdictional claims in published maps and institutional affiliations.

Open Access This article is licensed under a Creative Common C. Attribution 4.0 International License, which permits use, sharing, adaptation, distribution and reproduction in any medium or format, as long as you give appropriate credit to the original author(s) and the source, provide a link to the Creative Commons license, and indicate if changes were made. The images or other third party material in this article are included in the article's Creative Commons license, unless indicated otherwise in a credit line to the material. If material is not included in the article's Creative Commons license and your intended use is not permitted by statutory regulation or exceeds the permitted use, you will need to obtain permission directly from the copyright holder. To view a copy of this license, visit http://creativecommons. org/licenses/by/4.0/.

(c) The Author(s) 2020 